\title{
Introdução aos Sistemas de Recomendação para Grupos
}

\author{
Lucas A. M. C. Carvalho ${ }^{1}$ \\ Hendrik T. Macedo ${ }^{2}$
}

\begin{abstract}
Resumo: Sistemas de Recomendação tradicionalmente recomendam itens para usuários individuais. Em alguns cenários, entretanto, a recomendação para um grupo de indivíduos é mais adequada. Não existe ainda um bom volume de trabalhos científicos voltados para os chamados Sistemas de Recomendação para Grupos. Um dos grandes desafios desses sistemas é, por exemplo, como lidar adequadamente com as preferências de cada integrante do grupo para geração de recomendação. Com o intuito de contribuir para a evolução das pesquisas relacionadas aqui no Brasil, este tutorial trata esta e outras questões relacionadas ao tema. Após apresentar e discutir vários aspectos importantes, tais como, classificações, principais problemas, estratégias de agregação de preferências individuais, formas de avaliação, abordagens alternativas em curso e recomendação de sequência de itens, este tutorial contribui ainda ao propor uma nova estratégia de agregação e proceder a todo o processo de experimentação adequado, incluindo uma avaliação comparativa com as estratégias mais estabelecidas da literatura.
\end{abstract}

Palavras-chave: Sistemas de Recomendação para Grupos, Estratégias de Agregação, Formas de avaliação.

\begin{abstract}
Recommendation Systems usually recommend items to individual users. In some scenarios, however, the recommendation to a group of individuals is much more appropriate. There aren't, however, a good amount of scientific work focused on the so-called Recommender Systems for Groups. One of the great peculiarities of these systems is, for example, how to properly deal with the preferences of its members in order to provide good recommendations to the group. In order to attempt to fill this gap and to contribute to research maturation of the field here in Brazil, this tutorial covers this and other issues related to the theme. After presenting and discussing several important aspects, such as different categorizations, main issues, aggregation strategies for individual preferences, evaluation methods, alternative approaches and recommendation of sequence of items, this tutorial also contributes in proposing a new aggregation strategy and undertaking all appropriate experimentation process, including benchmarking with mainstream aggregation strategies.
\end{abstract}

Keywords: Recommender Systems for Groups, Aggregation Strategies, Evaluation methods.

\footnotetext{
${ }^{1}$ Núcleo de Pós-Graduação em Ciência da Computação, UFS

\{lucasaugustomcc@gmail. com\}

${ }^{2}$ Departamento de Computação, UFS

\{hendrikeufs.br\}
} 


\section{Introdução}

Sistemas de recomendação tradicionais realizam sugestões personalizadas de itens ainda não avaliados pelo usuário e que são de potencial interesse para o mesmo [67, 14, 1, 19]. Para que seja possível um sistema de recomendação gerar uma sugestão de item para um usuário, deve ser construído um perfil de interesse individual a partir da avaliação de itens feita por este. Com base nesse perfil de interesse, técnicas de análise de similaridade entre usuários ou itens, conhecidas como filtragem colaborativa [33] ou entre itens, e filtragem baseada em conteúdo [57] são usadas para geração da recomendação.

Além dessas técnicas tradicionais, novas abordagens para geração de recomendação para indivíduos vêm sendo propostas. Entre elas podemos citar as baseadas em personalidade [54, 34], baseadas em contexto [2], baseadas em conhecimento [43], baseadas em dados demográficos [74] e as baseadas em metadados [25].

A literatura científica relacionada a recomendações para indivíduos é de fato relativamente vasta. Entretanto, alguns cenários demandam recomendações para grupo de indivíduos e as técnicas acima citadas não resolvem este novo problema. Soluções que consideram grupos de indivíduos se tornarão essenciais à medida que os computadores migram dos desktop para o dia-a-dia das pessoas na forma de ambientes inteligentes e aplicações de computação ubíqua. São cenários potenciais para recomendação para grupos, por exemplo, a recomendação de repertório de músicas para uma festa, a recomendação de um restaurante para um almoço de negócios, um destino de viagem para a família e filmes para um grupo de amigos. Aos sistemas que geram sugestões com capacidade de satisfazer um grupo de usuários com interesses potencialmente conflitantes, denominamos Sistemas de Recomendação para Grupos [45].

Dentre os domínios aos quais uma recomendação para grupos pode se adequar muito bem, destacamos: filmes [55], turismo [35, 51, 31], música [48, 23, 75, 61, 20], programas de televisão [44, 78, 71], restaurante [51, 76], páginas da Web [40, 73], avisos em um espaço público [59], museu [37] e comunidades online [38]. Em [30], por exemplo, os autores propõe um sistema de recomendação para grupos para opções de turismo enquanto que em [22], os autores apresentam dois sistemas de recomendação de entretenimento para grupos de usuários, um para recomendação de músicas e outro para recomendação de filmes. Cabe ressaltar que, independentemente do domínio, as características do contexto em que a recomendação será feita é que são determinantes para a aplicabilidade da recomendação para grupos: nos últimos dois sistemas citados acima, por exemplo, a recomendação é gerada levando-se em consideração de que, seja a música ou o filme, este deverá ser apreciado por necessariamente mais de um usuário.

A Tabela11 relaciona os principais sistemas de recomendação para grupos já desenvolvidos e o domínio de aplicação de cada um. 
Tabela 1. Exemplos de Sistemas de Recomendação para Grupos

\begin{tabular}{ll}
\hline Título & Domínio \\
\hline MusicFX [48] & música \\
Let's Browser [40] & páginas web \\
GroupCast [49] & notícias \\
PolyLens [55] & filmes \\
Pocket Restaurant Finder [47] & restaurante \\
Intrigue [5] & turismo \\
Travel Decision Forum [35] & turismo \\
Adaptive Radio [20] & estações de rádio \\
I-SPY's [73] & páginas web \\
In-Vehicle Multimedia [80] & multimídia \\
YU's TV Recommender [78] & programas de televisão \\
CATS [50] & turismo \\
GroupFun [60] & música \\
jMusicGroupRecommender [22] & música \\
jMoviesGroupRecommender [22] & filmes \\
GroupMender [39] & notícias \\
MyPopCorn[16] & filmes \\
\hline
\end{tabular}

Neste tutorial, será apresentado o panorama atual das pesquisas sobre recomendação para grupos, organizado por diversos importantes e peculiares aspectos. Na seção 2, começamos por introduzir o conceito de grupo no contexto dos sistemas de recomendação. A seguir, apresentamos alguns dos diferentes eixos de classificação utilizados pela literatura corrente na seção 3 Na sequência, enumeramos as grandes problemáticas envolvidas na geração das recomendações na seção 4 Uma das grandes diferenças entre sistemas de recomendação individuais e para grupos é a necessidade de escolha de uma estratégia de agregação. Dedicamos a seção 5 para apresentação e discussão deste aspecto. Na seção 6, discutimos como uma abordagem alternativa baseada na Teoria dos Jogos pode lidar com estas escolhas de estratégias de agregação, em particular para grupos que são formados de forma aleatória. A seguir na seção 7, discutimos sobre a importância de se considerar a recomendação de sequência de itens. Finalmente, na seção 8, as dificuldades de se avaliar esses sistemas são tratadas e no sentido de tentar contribuir para a definição de uma metodologia para avaliação de sistemas de recomendação para grupos, que ainda são bem incipientes, propomos uma nova estratégia de agregação e realizamos a respectiva avaliação de qualidade ao comparar seu desempenho com seis das mais importantes da literatura corrente. 


\section{O conceito de Grupo}

Para a Sociologia, um grupo é um sistema de relações sociais, de interações recorrentes entre pessoas [28]. Também pode ser definido como uma coleção de várias pessoas que compartilham certas características, interagem uns com os outros, aceitam direitos e obrigações como sócios do grupo e compartilham uma identidade comum - para haver um grupo social, é preciso que os indivíduos se percebam de alguma forma afiliados ao grupo.

Neste trabalho adotamos o conceito geral de que grupo é um conjunto de duas ou mais pessoas reunidas por alguma particularidade, seja o mesmo local, uma mesma necessidade ou um mesmo interesse. Não necessariamente, os membros do grupo precisam ter todas essas particularidades simultaneamente.

Para sistemas de recomendação para grupos, quatro definições diferentes podem ser consideradas [13, 12]:

- Grupo estabelecido: um número de pessoas que explicitamente escolheram ser parte de um grupo, devido a algum interesse de longo prazo compartilhado;

- Grupo ocasional: um número de pessoas que, ocasionalmente, realizam algo juntos. Os seus membros têm um objetivo comum em um momento particular;

- Grupo aleatório: um número de pessoas que compartilham um mesmo ambiente em um momento particular, sem interesse explícito que as una;

- Grupo identificado automaticamente: grupos que são automaticamente detectados considerando as preferências dos usuários e/ou recursos disponíveis.

No sistema PolyLens [55], os autores definem os grupos apenas como permanentes e provisórios e utilizam mais características dos grupos: se o grupo é público e acessível a todos, ou privado e conhecido apenas pelos membros do grupo. Os autores perceberam que a persistência dos grupos é um problema relacionado tanto aos padrões de uso dos grupos pelos usuários quanto à privacidade. A privacidade torna-se crítica, por exemplo, no momento do envio de um convite para o usuário fazer parte do grupo e na exibição da previsão de preferência do usuário na recomendação para o grupo utilizada na explicação da recomendação do sistema.

\section{Classificação dos Sistemas de Recomendação para Grupos}

Masthoff [45] classifica os sistemas de recomendação para grupos segundo algumas dimensões: 
- Preferências individuais são conhecidas ou desenvolvidas com o tempo: na maioria dos cenários, o sistema de recomendação para grupos inicia com as preferências individuais. No CATS [50], excepcionalmente, as preferências individuais são construídas com o passar do tempo com a utilização de uma abordagem baseada em críticas.

- Itens recomendados são experimentados pelo grupo ou apresentados como opção: os membros do grupo podem experimentar uma música, como no MusicFX [48] ou receber uma lista de recomendação de filmes, como no PolyLens [55], que os membros do grupo podem desejar assistir.

- O grupo é ativo ou passivo: na maioria dos cenários, o grupo não influencia na maneira com que as preferências individuais são agregadas. Porém, no Travel Decision Forum [35] e CATS [50], o grupo pode negociar o modelo do grupo.

- Recomenda-se um único item ou uma sequência: na maioria dos cenários, pode-se recomendar apenas um único item como no caso de filmes, visto que pessoas normalmente assistem a apenas um filme por noite. Porém, também é possível a recomendação de um conjunto de itens para serem utilizados em sequência, mais usual, por exemplo, 'uma grade de programação de TV.

Segundo [15], mais duas dimensões podem ser adicionadas à classificação:

- Como as previsões de preferência individuais são obtidas: distingue-se principalmente entre filtragem colaborativa e baseada em conteúdo.

- Se são agregadas as recomendações ou perfis: no primeiro, as recomendações são produzidas para indivíduos e depois agregadas na recomendação para o grupo. No segundo caso, as preferências individuais são agregadas no modelo do grupo, e este modelo é usado para produzir a recomendação do grupo.

\section{Problemas relacionados aos Sistemas de Recomendação para Grupos}

A mudança de foco de um indivíduo para um grupo faz mais diferença do que se pode perceber à primeira vista. Em seus trabalhos, Jameson [35] e Jameson e Smyth [36] discutem os problemas mais importantes que surgem em sistemas de recomendação para grupos, organizando-as em termos de quatro subtarefas: (i) elicitação de informações de preferências do usuário, (ii) geração de recomendação, (iii) explicação da recomendação e (iv) suporte à tomada de decisão final pelos usuários. Além destas, questões relacionadas à privacidade dos membros de um grupo são também novos problemas a serem considerados. A seguir, cada um destes problemas são discutidos. 


\subsection{Elicitação de informações de preferências do usuário}

A maioria dos métodos de elicitação de preferência dos usuários utilizados em recomendação para indivíduos também podem ser utilizados na recomendação para grupos. As preferências do usuário podem ser obtidas de forma direta, no método conhecido como avaliação explícita, ou de forma indireta, inferindo as preferências através das ações ou feedback do usuário, no método conhecido como avaliação implícita. A avaliação implícita é menos comum, porém pode ser encontrada em Senot et al. [71] e Lage, Durão e Dolog [39]. O trabalho de Senot et al. [71] infere as preferências dos usuários baseado no tempo que cada indivíduo gasta assistindo a um filme, por exemplo. Quanto mais uma pessoa assiste a um filme, mais o autor assume que ele está interessado no mesmo. Em Lage, Durão e Dolog [39], os autores inferem as preferências dos usuários através das postagens deste usuário em uma rede de microblogging.

A importância desta tarefa em recomendação para grupos, é que cada membro pode ter algum interesse em conhecer as preferências dos outros membros para, entre outras coisas, persuadir um usuário a realizar uma avaliação similar à dele. Algumas vezes, não desejando que sua preferência seja minoritária, a preferência do usuário corrente depende em parte da preferência e/ou antecipação do comportamento de um ou mais outros membros do grupo. Alternativamente, um membro pode desejar conhecer a preferência dos outros membros para simplesmente procurar minimizar conflitos que poderiam tornar difícil para o grupo encontrar uma solução. No Travel Decision Forum, o membro corrente pode copiar todas ou parte das preferências de um outro membro para evitar esforço ou realizar a aquisição de um conhecimento que o membro corrente não possui [35]. É possível ainda realizar uma cópia automática das preferências de outro usuário do sistema que seja similar ao novo usuário de um sistema de recomendação para grupos, e assim tentar enfrentar o problema que um novo usuário tem quando ainda possui poucas avaliações realizadas, e o sistema não consegue gerar uma previsão de preferência para este usuário [63].

Enquanto a maioria dos trabalhos utiliza preferências positivas, isto é, o usuário avalia itens dos quais ele gosta, o Adaptive Radio [20] utiliza preferências negativas, ou seja, o usuário informa que não tem interesse em determinado item e que este não deve ser considerado em uma eventual recomendação. Os autores defendem que adotar preferências negativas torna menos entediante a avaliação dos itens para o usuário, visto que o sistema assume que o usuário gosta de todos os itens disponíveis, a menos que o usuário o avalie negativamente. $\mathrm{O}$ autor ainda afirma que sistemas de preferência negativa podem aumentar a influência dos membros do grupo que possuem opiniões minoritárias. Em Chao [20], a abordagem de preferências negativas pode reduzir o processo de conformação que suprime as opiniões minoritárias exigindo que o grupo alcance um consenso, o que pode aliviar a necessidade da minoria ter de aceitar a opinião da maioria, e permite tornar o processo de tomada de decisão anônimo, o que, por sua vez, reduz a pressão social exercida pela maioria. Por tanto, por 
ajudar a fortalecer a opinião das minorias, as avaliações negativas assumem um papel mais importante em sistemas de recomendação para grupo do que para sistemas de recomendação para indivíduos.

\subsection{Geração de recomendação}

A geração de recomendação é a tarefa mais estudada pelos pesquisadores em recomendação para grupos, visto que para gerar uma recomendação, alguma estratégia de agregação precisa ser utilizada e a escolha de qual estratégia se utilizar não é uma tarefa trivial. As informações sobre as preferências individuais devem ser combinadas de uma forma que o sistema possa verificar a adequação de itens particulares para o grupo como um todo.

Apesar das várias abordagens de agregação diferirem na maneira de manipular e representar as preferências dos usuários, praticamente todas fazem uso de um dos três esquemas: (i) agregação de recomendações individuais, (ii) construção de modelo único de preferências do grupo e (iii) agregação das avaliações/preferências para itens particulares.

4.2.1 Agregação de recomendações individuais A agregação de recomendações individuais é um método simples de agregação que, a partir da geração de uma lista com um pequeno número de recomendações para cada membro, mescla estas listas em uma única lista. Apesar de ser a estratégia menos adotada em recomendação para grupos, ela é uma das estratégias utilizadas no Polylens [55], no Intrigue [5], com subgrupos de pesos diferentes, e em [4], com uma função de consenso que combina a relevância dos itens para o usuário e o desacordo entre os membros. Ela também é a estratégia analisada em [8], com o uso de agregação de listas (rank aggregation), que produz um único ranking de itens a partir de diferentes ranking de itens. Esta estratégia agrada a maioria dos membros do grupo, porém pode não satisfazer a todos. Para este fim, deve ser adotada uma técnica auxiliar, por exemplo, a eliminação dos itens menos importantes, ou seja, de acordo com a relevância do item para cada membro do grupo [38].

Uma metodologia de negociação cooperativa para a solução do problema é apresentada no trabalho de Bekkerman [9]. Cada usuário é representado por um agente negociador, onde todos os agentes possuem o mesmo comportamento. Uma vez que as recomendações individuais são obtidas, o processo de negociação se inicia, onde dentro de uma negociação o agente pode aceitar ou recusar uma oferta.

4.2.2 Construção de modelo de preferência do grupo Esta estratégia de agregação de construção de um modelo único de preferência do grupo não utiliza a predição da avaliação de usuários, mas apenas as informações de preferência individual dos membros do grupo para alcançar um modelo de preferências para o grupo como um todo. O modelo do grupo é 
utilizado para a previsão da avaliação dos itens candidatos à recomendação para o grupo. Em alguns casos, o modelo de preferência do grupo pode ser visualizado como uma agregação dos modelos de preferências individuais, como no caso do Let's Browser [40], IN-VEHICLE Multimedia [80] e YU's TV Recommender [78]. Em outros casos, o modelo de preferência de grupo pode ser representado como uma agregação de modelos de preferência de subgrupos, como ocorre no sistema Intrigue [5], que foi projetado para auxiliar guias turísticos na criação de roteiros turísticos para grupos heterogêneos que incluem subgrupos relativamente homogêneos (ex. crianças e idosos). Outros sistemas que utilizam essa estratégia são o I-SPY'S [73] e Travel Decision Forum [35].

Construir um modelo único de preferência para o grupo é vantajoso quando os membros do grupo terão a oportunidade de examinar e/ou negociar sobre esse modelo antes ou depois que ele tenha sido verdadeiramente aplicado. O desconhecimento dos critérios e parâmetros utilizados para definir este único modelo que representaria o grupo como um todo pode levar à desconfianças por parte dos membros deste grupo acerca da qualidade e efetividade do modelo. Ao contrário, se é dado a chance de se examinar e se discutir o modelo antes de sua aplicação e, sobretudo, de se revisitar suas características após análise conjunta dos efeitos de sua aplicação, a confiança dos membros em sua efetividade será bem maior.

Uma desvantagem de se utilizar um único modelo para o grupo é que estes algoritmos podem produzir recomendações que satisfazem muitos mas não todos os membros de um grupo, o que pode não corresponder à função de valor social utilizada. E ainda, a predição do grupo pode estar fora do alcance de qualquer predição individual para os membros do grupo, o que pode ser desorientante para os membros ou tornar difícil a explicação da recomendação [35].

Enquanto a maioria dos trabalhos utiliza a avaliação ou preferência dos itens como um todo, YU's Recommender mescla as preferências individuais dos usuários para as características de um programa de televisão (por exemplo, gênero, ator, e palavras-chave) e não avaliações individuais para estes programas.

Em sistemas que utilizam a preferência negativa para encontrar as preferências do grupo, é possível considerar a união das preferências negativas dos indivíduos. Este conjunto de preferências combinadas filtrará os itens que não agradam a nenhum membro do grupo. A solução restante é chamada de solução de consenso, pois esses itens foram aprovados implicitamente por todos. Apesar desta ser formalmente equivalente a encontrar a interseção das preferências positivas do mesmo grupo, a abordagem pode ser mais efetiva na prática [20].

Devido ao conhecimento das preferências (positivas ou negativas) do usuário ser tipicamente incompleto ou incerto, um esquema de preferências individuais positivo, provavelmente subestimaria o número de soluções que um usuário toleraria, enquanto o esquema 
negativo superestimaria este valor. Devido à interseção das preferências de múltiplos usuários ser difícil de encontrar, caso existam de fato (problema encontrado no GroupCast [49]), Chao [20] acredita ser prefererível errar na superestimativa do que na subestimativa do espaço de soluções aceitáveis.

Uma tendência para geração do modelo de preferência do grupo vem sendo estudada a partir do uso da personalidade dos membros do grupo [66, 64, 41, 26, 62, 65]. Este método com uso da personalidade é utilizado basicamente para minimizar o problema do coldstart [68] para novos usuários, onde a recomendação é gerada para itens daqueles usuários mais antigos com alta similaridade de personalidade com o usuário novo.

\subsubsection{Agregação das avaliações/preferências para itens particulares Pode utilizar a} predição da avaliação de itens ainda não avaliados ou a avaliação explícita dos itens. A avaliação explícita é estratégia mais utilizada e os seus diversos métodos encontrados na literatura serão apresentados na seção 5 .

Este tipo de estratégia tem diversas vantagens. Elas apresentam resultados que podem ser diretamente relacionados aos resultados que seriam percebidos por membros individuais do grupo. Isto significa que os resultados são relativamente fáceis de explicar. Entretanto, as recomendações são menos propensas a identificar itens inesperados para os membros do grupo.

A maior diferença entre as estratégias é a ênfase colocada na satisfação dos indivíduos - particularmente, evitar misery - comparada à satisfação da maioria do grupo. O problema conhecido como misery ocorre quando um item com baixa avaliação, de acordo com as preferência de um membro do grupo, é recomendado para o grupo, deixando este membro bastante insatisfeito.

Alguns trabalhos vem realizando uma análise da interação entre os membros do grupo considerando a correlação entre os mesmos [41, 11]. O objetivo é prever um peso para cada membro, para ser utilizado juntamente com as avaliações individuais na geração da recomendação para o grupo.

Wang, Liu e Zhao [77] buscam analisar fatores sociais como relações de confiança (trust) como fator no modelo de predição para o grupo, buscando encontrar a influência dos seus membros e considerando a relação entre vários usuários no grupo. O autor procura otimizar a função de agregação de acordo com as diferentes influências dos membros do grupo que ele afirma que pode melhor refletir suas características sociais. Em [64], QuijanoSánchez, Garcia e Díaz-Agudo também analisam a influência social no grupo a partir da personalidade dos seus membros que podem ser mais ou menos cooperativos com relação ao conflito para escolha dos itens a serem recomendados. Em outro trabalho [65], QuijanoSánchez et al. apresentam uma abordagem em que usuários mais influentes desejam criar 
alianças com outros usuários. Estas alianças objetivam reunir um peso maior aos itens de preferência desses usuários mais influentes para que estes itens sejam recomendados para o grupo.

Outras estratégias são propostas utilizando votação e probabilidade [61,75]. Enquanto Popescu e Pearl [61] utilizam o voto do membro do grupo em uma música para aumentar a probabilidade desta ser escolhida para recomendação, Sprague, Wu e Tory [75] utilizam o voto para que cada membro do grupo escolha ao menos uma música a ser recomendada.

\subsection{Explicação da recomendação}

O objetivo da explicação não é necessariamente convencer os membros do grupo a aceitarem as recomendações do sistema. Pelo contrário, as explicações do sistema são geralmente melhor vistas como informações que colocam os membros do grupo em uma melhor posição para tomar uma decisão final, que pode até desviar radicalmente da recomendação feita.

É natural que membros do grupo desejem entender, até certo ponto, como a recomendação foi realizada e, em particular, o quão atrativo um item recomendado parece ser a cada membro do grupo. Com sistemas de recomendação para grupos é possível, em princípio, apresentar esta análise para cada membro, para o grupo como um todo, e talvez para subconjuntos de membros.

No Polylens [55], explora-se três modelos de organização das informações disponibilizadas para explicação das recomendações: uma interface somente do grupo, uma interface composta, e uma interface focada no individual.

$\mathrm{Na}$ interface somente do grupo, os itens são exibidos com a recomendação para o grupo. Estas interfaces evitam revelar as informações de preferência de outros membros do grupo, o que acaba evitando que os membros do grupo realizem o balanceamento com os interesses de outros membros para a seleção de itens.

Interfaces compostas exibem uma lista de filmes recomendados tanto com a previsão para o grupo quanto para membros individuais. Dependendo da política de privacidade do sistema, membros particulares do grupo podem ser omitidos da listagem. Estas interfaces permitem que membros do grupo façam o balanceamento da estimativa do bem-estar previsto para o grupo com a satisfação prevista para cada membro do grupo. Um trabalho recente de recomendação para grupos para elicitação de requisitos de software obteve melhores resultados de satisfação dos usuários ao não exibir as preferências individuais durante o início do processo de tomada de decisão pelo grupo [27].

Interfaces focadas no individual exibem itens baseados nas preferências individuais do usuário. Elas podem até inteiramente omitir a recomendação para o grupo, sendo que 
tal recomendação pode tanto ser exibida ou usada para filtrar os filmes sendo exibidos. O Polylens [55] implementa a interface composta para a exibição das recomendações com o interesse de oferecer o máximo de informações enquanto tenta minimizar a carga de informação para os usuários.

\subsection{Suporte à tomada de decisão final pelos usuários}

Se o recomendador para grupos é projetado a partir do pressuposto que há alguns aspectos do problema de decisão que são melhor tratados pelos próprios usuários, a função do sistema serve de suporte à decisão ao invés de tomada de decisão.

No suporte à decisão é assumido que os membros do grupo chegarão a uma decisão final através de discussão convencional, como ocorre no sistema Collaborative Advisory Travel System (CATS) [50], ou um membro do grupo é responsável por tomar a decisão final, como ocorre nos sistemas Let's Browser [40] e Intrigue [5].

Na tomada de decisão, o sistema simplesmente traduz a solução com a maior avaliação em ação, sem requisitar o consentimento de nenhum membro do grupo. O Adaptive Radio [20], Flytrap [23] e MusicFX [48] realizam a tomada de decisão pelo grupo, visto que não é praticável haver uma discussão para cada recomendação de música para o grupo.

A tarefa de suporte à tomada de decisão é a que menos tem atraído atenção de pesquisas, pois os sistemas geralmente deixam o próprio grupo chegar a um consenso sobre o item que será consumido a partir da lista de recomendação.

\subsection{Privacidade dos membros do grupo}

Sistemas de recomendação tradicionais exploram dados do indivíduo para gerar recomendações personalizadas. Ao tentar melhorar a qualidade das recomendações, esses sistemas tentam coletar o maior conjunto possível de dados do usuário alvo. Claramente, fica estabelecida uma contradição importante: ao mesmo tempo em que o usuário se satisfaz com recomendações altamente precisas aos seus gostos e peculiaridades, este se sente incomodado em desconhecer como tal sistema conhece tanto sobre suas preferências pessoais. Sistemas de recomendação devem, desta forma, utilizar de forma parcimoniosa os dados do usuário ao tempo que devem implementar soluções de segurança que evitem que estes dados pessoais coletados não possam ser facilmente acessados por outros usuários com intenções maliciosas.

Alguns trabalhos exploram diretamente estas questões de privacidade de usuário de sistemas de recomendação tradicionais [72], [21], [10], [52], [3]. Outra linha de trabalhos exploram questões sobre privacidade de usuários enquanto integrantes de redes sociais virtuais e, portanto, de certa forma, participantes de grupos de usuários [79]. Entretanto, muito pouco é discutido sobre as questões de privacidade específicas quando migramos a discussão para 
sistemas que recomendam para grupos de usuários.

Nestes casos, a privacidade torna-se crítica, por exemplo, no momento do envio de um convite para o usuário fazer parte de um determinado grupo e na exibição da previsão de preferência do usuário na recomendação para o grupo utilizada na explicação da recomendação do sistema.

O uso de modelos individualizados de preferência para recomendações para um grupo pode também gerar problemas relacionadas à privacidade. Uma vez que o modelo individual se torna de conhecimento do grupo, um determinado membro pode, por exemplo, se incomodar com a publicitação de seu interesse por filmes adultos.

\section{Estratégias de Agregação de Preferências Individuais}

Como comentado na seção 4.2 .3 , a necessidade de escolha de um método de agregação é a diferença mais estudada entre recomendação para grupos e recomendação para indivíduos. Nesta seção, é dado foco às técnicas de agregação das preferências para itens particulares.

Masthoff [44] concentra seus estudos nas estratégias de agregação de preferência individual baseadas na Social Choice Theory [6, 69, 58]. O problema relacionada à social choice ou group decision making é decidir o que é melhor para um grupo dado a opinião dos membros desse grupo.

As estratégias contempladas nos trabalhos de Masthoff [44, 45] e diversos outros trabalhos na literatura, Senot et al. [71] e [29], para citar mais alguns, são:

- Average: assume uma importância igual para todos os membros do grupo e computa a média da satisfação de todo o grupo para qualquer item dado. Pode assumir também diferentes pesos para os membros do grupo, como utilizado no Intrigue [5]. O YU's Recommender [78] também utiliza esta estratégia com algumas modificações. A desvantagem desta estratégia é que enquanto em grandes grupos, um membro pode sempre perder uma vez que sua opinião pode ser minoritária, em grupos pequenos, isto pode não ocorrer, pois a opinião de cada indivíduo terá um maior impacto na média.

- Least Misery: assume a avaliação do membro menos satisfeito com um item dado como o valor da satisfação para todo o grupo. O Polylens [55] utiliza esta estratégia de agregação. A desvantagem dessa estratégia é que um item em que todos os membros estão pouco satisfeitos, pode ser recomendado no lugar de um item em que apenas um usuário está muito insatisfeito e os demais muito satisfeitos, podendo assim ocorrer a misery para todo o grupo na recomendação. A vantagem é que um item que um membro do grupo esteja muito insatisfeito não será recomendado para os demais, podendo aumentar a confiança (trust) do sistema. 
- Most Pleasure: assume a avaliação do membro mais satisfeito com um item dado como o valor da satisfação para todo o grupo. A desvantagem dessa estratégia é que qualquer avaliação de um membro menos satisfeito com este item não terá nenhum efeito na recomendação. A vantagem é que um item muito bem avaliado, por ao menos um membro do grupo, pode ser recomendado e aumentar a descoberta de itens (serendipity) para o restante do grupo.

- Utilitarian Multiplicative: os valores das avaliações dos membros para um dado item são multiplicados e quanto maior esse valor, mais relevante o item será na lista de recomendação para o grupo. Possui a mesma desvantagem da estratégia Average. Esta estratégia apesar de pouco utilizada na literatura, teve bons resultados em uma pesquisa sobre a racionalidade das estratégias utilizadas por usuários reais para recomendação de uma sequência de itens para o grupo [44].

- Average Without Misery: gera uma nova lista de avaliações com as médias das avaliações individuais dos membros do grupo para um dado item, porém retira os itens que possuam qualquer avaliação de preferência abaixo de um valor mínimo. O MusicFX [48] utiliza esta estratégia de agregação.

- Plurality Voting: cada membro do grupo vota no item com maior preferência individual. Apesar da vantagem de satisfazer a maioria do grupo, ela eventualmente deixa a minoria totalmente insatisfeita.

- Borda count: a cada item na lista ordenada dos membros do grupo é atribuída uma pontuação que corresponde à sua posição na lista: a alternativa no final da lista recebe zero pontos, a próxima acima, um ponto, e assim por diante.

- Fairness: itens de maior preferência para cada um dos membros do grupo são selecionados. Quando itens são avaliados igualmente, a opinião dos outros é levada em consideração. A idéia por trás desta estratégia é que não é tão ruim assistir a algo que você detesta, considerando que você também assista a algo que você adore. Esta estratégia frequentemente é aplicada quando pessoas tentam dividir de forma justa um conjunto de itens: uma pessoa escolhe o primeiro, outra o segundo e assim por diante até todos terem feito uma escolha. Depois, todo mundo escolhe um segundo item, geralmente iniciando da pessoa que teve que escolher por último na rodada anterior. $\mathrm{E}$ continua até todos os itens terem sido escolhidos.

- Dictatorship ou Most respected person: a idéia desta estratégia é que grupos podem ser dominados por uma pessoa. Por exemplo, visitantes podem ter mais influência na decisão do que os habitantes da casa. Esta estratégia é utilizada com mais frequência com a técnica de filtragem colaborativa com o nome de "estratégia de vizinhança mais próxima": somente as preferências dos indivíduos com gosto mais próximos ao 
interessado pela recomendação são utilizados. Um uso mais sofisticado da diferença do estado social seria atribuir pesos a avaliações individuais. Esta estratégia também tem sido usada em filtragem colaborativa no sistema Intrigue [5] com base no uso da estratégia Average com pesos.

- Copeland Rule: esta é uma forma de voto pela maioria. Seu objetivo é ordenar as alternativas de acordo com o índice de Copeland: o número de vezes que uma alternativa supera outras alternativas menos o número de vezes que ela perde para outras alternativas.

- Approval Voting: eleitores são permitidos votar para quantas alternativas desejarem. A intenção é promover a eleição de alternativas moderadas, alternativas que não são fortemente rejeitadas.

De acordo com [70], essas estratégias podem ser divididas em três categorias (ver Tabela 2): (i) estratégias baseadas em consenso, que consideram as preferências de todos os membros do grupo; (ii) estratégias baseadas em maioria, que utilizam os itens mais populares entre os membros do grupo; (iii) estratégias borderline, que consideram somente um subconjunto de itens, em perfis individuais, baseados nas regras de usuários ou qualquer outro critério relevante. Entre as estratégias nesta categoria estão a estratégia Dictatorship que usa somente a preferência de um único membro, o qual impõe seu gosto para o resto do grupo. As estratégias Least Misery e Most Pleasure mantêm para cada preferência, respectivamente, o menor e o maior nível de interesse entre os membros do grupo.

Tabela 2. Categorias das estratégias de agregação individual [Senot et al., 2011]

\begin{tabular}{l|l|l}
\hline Consenso & Maioria & Borderline \\
\hline $\begin{array}{l}\text { average, average } \\
\text { without misery, multipli- } \\
\text { cative, fairness }\end{array}$ & $\begin{array}{l}\text { vlurality voting, appro- } \\
\text { val voting, Copeland }\end{array}$ & $\begin{array}{l}\text { least misery, most plea- } \\
\text { rule, borda count }\end{array}$ \\
\hline
\end{tabular}

\subsection{Exemplo ilustrativo das estratégias de agregação}

Sendo a Tabela 3 preenchida com as avaliações/preferências individuais de cada um dos membros do grupo, a Tabela 4 utiliza essas avaliações/preferências para exemplificar as estratégias de agregação. 
Tabela 3. Preferências individuais.

\begin{tabular}{lcccccccc}
\hline & A & B & C & D & E & F & G & H \\
\hline \hline Rafael & 5 & 10 & 3 & 10 & 5 & 10 & 6 & 10 \\
\hline Joana & 10 & 4 & 6 & 2 & 1 & 9 & 6 & 8 \\
\hline Paula & 5 & 5 & 9 & 4 & 6 & 8 & 5 & 3 \\
\hline
\end{tabular}

\subsection{Critérios de Qualidade}

Cada estratégia apresentada possui vantagens e desvantagens e a comparação entre elas necessita de critérios de qualidade que podem ser resumidos em seis pontos [36], sendo que os pontos em que este tutorial se concentra são: maximização da satisfação média, minimização da misery e assegurar algum grau de justiça. A escolha destes três critérios deve-se a percepção de uma maior facilidade, comparado aos outros critérios não selecionados, de realizar uma estimativa para o cálculo de cada um desses critérios baseado nas preferências dos membros do grupo e na recomendação proposta para o grupo.

1. Maximizar satisfação média: pode ser alcançada por uma função de agregação que computa algum tipo de média das previsões de satisfação de cada membro para usar como base de seleção dos itens candidatos.

$$
R_{i}=\operatorname{average}\left(r_{i j}\right)=1 / n \sum r_{i j}
$$

Na Equação 1, $r_{i j}$ é a avaliação do usuário $j$ para o item $i, n$ é a quantidade de membros no grupo e $R_{i}$ é a avaliação do grupo para o item $i$, segundo o critério adotado.

O Pocket Restaurant Finder [47] aplica uma variante desta fórmula para as previsões de avaliações de restaurantes pelos membros do grupo que estão se preparando para sair para jantar juntos.

2. Minimização da misery: deve evitar uma solução que deixe um ou mais membros muito insatisfeitos com a recomendação. Mesmo o membro do grupo mais egocêntrico pode não desejar interagir com outro membro que está claramente insatisfeito; e este membro pode recusar continuar com a solução em qualquer caso. Isto significa levar em conta este fator como uma restrição que deve ser atendida pela solução: a menor previsão de avaliação de um item não pode ser menor do que um dado valor de corte. No PolyLens [55], um dos critérios aplicados é a minimização da misery através da Equação 2.

$$
R_{i}=\min r_{i j}
$$


Tabela 4. Estratégias de agregação de preferência individual (adaptado de [Masthoff, 2004].

\begin{tabular}{|l|l|}
\hline Estratégia & Exemplo \\
\hline Plurality Voting & $\begin{array}{l}\text { F é escolhido primeiro, pois tem a maior avaliação pela } \\
\text { maioria do grupo, seguido por H (que tem a maior avali- } \\
\text { ação da maioria quando excluindo F). }\end{array}$ \\
\hline Average & avaliação para F é 6, pois (10+9+8)/3=9 \\
\hline Multiplicative & a avaliação para H é 240, pois 10*8*3. \\
\hline Borda Count & $\begin{array}{l}\text { A avaliação para A é 8, pois 1 (compartilhado penúltimo } \\
\text { para Rafael) + 7 (primeiro para Joana) + 0 (último para } \\
\text { Paula) }\end{array}$ \\
\hline Copeland Rule & $\begin{array}{l}\text { A avaliação para F é 7, como F supera 7 itens } \\
\text { (A,B,C,D,E,G,H) e não perde para nenhum item. }\end{array}$ \\
\hline Approval Voting & A avaliação para C é 1 e de B é 1. \\
\hline Least Misery & A avaliação para D é 2, pois é o menor entre 10, 2 e 4. \\
\hline Most Pleasure & A avaliação para H é 10, pois é o maior entre 10, 9 e 3. \\
\hline Average without Misery & $\begin{array}{l}\text { A avaliação de C é 6 (a média de 3,6,7), enquanto E é } \\
\text { excluído por que Joana detesta ele. }\end{array}$ \\
\hline Fairness & $\begin{array}{l}\text { Item A pode ser escolhido primeiro (melhor para Joana), } \\
\text { seguido de C (melhor para Paula) e B (entre os melhores } \\
\text { para Rafael). }\end{array}$ \\
\hline Most respected person & $\begin{array}{l}\text { Se Joana é a pessoa mais respeitada, então a avaliação do } \\
\text { grupo para E é 1. Se Paula é a mais respeitada, então é 6. }\end{array}$ \\
\hline
\end{tabular}

3. Assegurar algum grau de justiça: uma solução que satisfaz todo mundo igualmente bem é em geral preferível a uma que satisfaz alguns em detrimento dos outros. No caso de minimização da misery, o objetivo de assegurar justiça é em geral combinada com algum outro critério, pois ninguém deseja uma solução perfeitamente justa que leve todo mundo a ficar igualmente insatisfeito. No cálculo da justiça, pode-se assumir uma importância igual para todos os membros do grupo e computar a média da satisfação de todo o grupo para qualquer item dado e uma penalidade para a variação das avaliações do item pelos membros do grupo. Quanto maior a variação das avaliações, maior a penalidade.

$$
R_{i}=\operatorname{average}\left(r_{i j}\right)-w \times \operatorname{stddev}\left(r_{i j}\right),
$$

Na Equação 3, $w$ é o peso que reflete a importância da justiça e a função stddev consiste no desvio padrão entre as avaliações para o item $i$. 
4. Tratar diferente membros do grupo quando apropriado: em certas situações geralmente é aceito que a preferência de alguns membros do grupo (crianças e idosos, principalmente) sejam tratados diferentemente dos demais, recebendo um peso maior.

No Intrigue [5], o guia de turismo é capaz de definir pesos maiores para subgrupos de pessoas com necessidades especiais ou crianças, assumindo que estes grupos são menos propensos a aceitar soluções mesmo que parcialmente insatisfatórias para eles.

5. Desencorajar a manipulação do mecanismo de recomendação: retirar a motivação para o usuário votar de forma não verdadeira nos itens candidatos com a intenção de aumentar as chances de recomendação de um item específico.

6. Assegurar compreensão e aceitabilidade: membros do grupo algumas vezes querem ser capazes de entender a lógica de funcionamento que gerou a recomendação. Eles também podem desejar verificar em que medida os critérios anteriores estão sendo cumpridos.

\section{Recomendação baseada na Teoria dos Jogos}

Em grupos que são formados de forma aleatória, considera-se que os usuários possuem interesses próprios, o que significa que o usuário tem sua própria definição de cada item que ele gostaria de receber como recomendação. Um exemplo atual que fomentará a formação de grupos deste tipo são os grandes eventos esportivos [56] que irão ocorrer no Brasil durante a Copa do Mundo de 2014 e nos Jogos Olímpicos e Paralímpicos de 2016. As milhares de pessoas presentes certamente formarão grupos aleatórios e heterogêneos que, potencialmente serão alvo de recomendações de músicas, informações ou notícias, restaurantes, shows, entre outros.

O equilíbrio de Nash, da teoria dos jogos [53], trata da resolução de forma racional de conflitos de interesses por jogadores de uma situação modelada como um jogo. Sendo um perfil de estratégia estável, o equilíbrio significa que considerando que os demais jogadores não irão modificar suas próprias estratégias, o jogador corrente não possui qualquer incentivo para modificar a sua. A teoria dos jogos procura encontrar estratégias racionais em situações em que o resultado depende não só da própria estratégia do jogador, mas também das estratégias escolhidas por outros jogadores que possivelmente possuem estratégias diferentes.

A resolução desse tipo de conflito com uma abordagem cooperativa em grupos aleatórios e heterogêneos pode resultar em uma negociação em que não se chegue a um acordo quando os membros do grupo possuem interesses próprios como ocorre em [9, 32].

Em [18], os autores mostram que a modelagem da estratégia de recomendação para grupos como um jogo não-cooperativo da Teoria dos Jogos pode atender à necessidade de um equilíbrio entre satisfazer as próprias preferências do jogador, isto é, do membro do grupo, 
e evitar a insatisfação dos demais membros do grupo através da justiça na recomendação para o grupo. Apesar dos usuários possuírem interesses próprios, sempre haverá ao menos um equilíbrio de Nash, ou seja, o sistema sempre poderá realizar uma recomendação para o grupo.

Na questão relacionada à formação dos grupos, fase anterior à recomendação, os trabalhos relacionados em geral não apresentam uma preocupação com o bem-estar social total do grupo formado ou a utilização de uma função de utilidade para determinar o melhor grupo a ser formado; é apenas considerado alguma função de similaridade entre os membros do grupo. Em [17], os autores contornam esta situação mostrando que a formação dos grupos pode ser modelada como um problema de geração de estruturas de aliança em um jogo cooperativo de forma a maximizar o bem-estar social total (Social Welfare).

\section{Sequência de itens}

Em alguns cenários, como recomendação de grade de programação de TV ou repertório musical em uma festa, pode ser interessante a recomendação de diferentes itens para serem utilizados em sequência. Este tipo de recomendação consiste em resolver o problema de múltiplas decisões que a utilização de mais de um item gera para um grupo de usuários. Entre os trabalhos que recomendam sequência de itens podemos destacar o YU's Recommen$\operatorname{der}$ [78] e [44] com recomendação de programas de TV, um recomendador baseado em casos [7], [48], Adaptive Radio [20] com recomendação de músicas e Intrigue [5] com atrações turísticas.

A recomendação de sequência possui alguns problemas que não são considerados em recomendações únicas, como a mudança dos membros do grupo e a ordem dos itens. Por exemplo, na recomendação de uma sequência de filmes para um grupo, a ordem em que os filmes devem ser vistos, pode influenciar na satisfação do grupo. É importante compensar usuários que tiveram a satisfação baixa no filme anterior. Também deve-se saber como lidar com a recomendação quando um dos membros deixa o grupo e a sua preferência havia influenciado significativamente nos filmes seguintes da sequência.

Em grupos estabelecidos, geralmente as recomendações não são fornecidas de maneira $a d$-hoc, mas os usuários tem uma interação prolongada com o sistema. É importante também nesses casos, mesmo que o objetivo seja uma única recomendação, os membros cuja satisfação tenha sido baixa nas recomendações anteriores sejam compensados na próxima recomendação [11].

A recomendação de sequência pode ser tratada em três abordagens [36]: decisões independentes, desconsiderando a recomendação como uma sequência; recomendação de sequência como uma única decisão; ou decisões independentes, porém considerando a reco- 
mendação como uma sequência. Estas abordagens serão descritas a seguir.

\subsection{Decisões independentes, desconsiderando a sequência}

Esta é a abordagem mais simples e que ignora o fato que a recomendação consiste em uma sequência de decisões. Devido à sua simplicidade, esta abordagem tende a ser computacionalmente simples e fácil de explicar para os usuários. Uma desvantagem é que o objetivo de assegurar justiça entre os membros do grupo somente é considerada com respeito a decisões individuais, enquanto poderia ser vantajoso considerar a justiça no conjunto completo de decisões.

Como exemplo, pode parecer justo recomendar um único programa de TV que seja menos atraente para um membro específico do grupo do que para os demais membros, se a satisfação geral deste membro do grupo com a sequência de programas seja comparável ao dos demais membros deste grupo.

O YU's Recommender adota esta abordagem, e o autor ainda afirma que ela difere das demais abordagens, pois enquanto as outras tentam não deixar ninguém no grupo muito insatisfeito (evitar misery), a sua abordagem recomenda um programa de TV por vez e se preocupa em deixar a maioria do grupo satisfeita.

\subsection{Recomendação única}

Esta abordagem é aplicável somente se é conhecido previamente qual sequência particular de decisões deve ser feita e quais membros do grupo estarão envolvidos (por exemplo, quantas vezes e em quais ocasiões específicas um grupo irá jantar junto). Esta abordagem permite que seja direta a aplicação de critérios como justiça para a sequência completa.

Porém, nesta abordagem não é possível considerar o resultado de decisões anteriores, visto que o processo de tomada de decisão para a sequência inteira é finalizado antes que qualquer decisão seja tomada. Também, os procedimentos computacionais necessários tendem a ser mais complexos; por exemplo, o número de sequências possíveis pode ser muito grande e inviabilizar que um sistema possa iterar sobre todas as possíveis sequências para avaliar suas adequações para o grupo.

\subsection{Decisões independentes, considerando a sequência}

Nesta abordagem, as decisões são tomadas considerando as anteriores e podem utilizar um critério para ser considerado na recomendação subsequente. Por exemplo, para cada decisão individual pode ser abordado com o objetivo de maximizar a satisfação geral dos membros do grupo. Entretanto, se o sistema perceber que, a partir de um dado momento, um 
membro do grupo tem estado menos satisfeito que os demais, pode ser dado a este membro um maior peso nas decisões subsequentes, até que a discrepância entre a sua satisfação e a dos demais tenha sido eliminada. Esta abordagem é capaz de considerar os atuais resultados das decisões (ao contrário de somente os resultados das previsões, como na abordagem anterior).

Apesar de mais complexo que a abordagem de decisões independente, o método pode ser explicado razoavelmente se ele corresponder com esquemas de tomada de decisão familiares ao dia a dia das pessoas. Esta abordagem não requer um conjunto de decisões conhecido previamente, porém ele pode ser aplicado mais efetivamente se uma boa proposta é conhecida. Por exemplo, se o sistema decide conceder uma certa dose extra de satisfação para um membro particular do grupo na geração da recomendação subsequente, para isso pode ser desejável saber quantas decisões restam ser tomadas.

Um exemplo desta abordagem pode ser encontrada no sistema de seleção de música do Flytrap [23]. O sistema escolhe músicas uma de cada vez porque o conjunto de membros presentes para ouvir muda frequentemente. Entretanto, o procedimento de seleção considera restrições impostas por um agente DJ que tenta, por exemplo, evitar mudanças abruptas e de gênero que provoquem distrações.

Um outro trabalho também utiliza esta abordagem para recomendar programação para canais de música, cuja sequência de canções possuem como critério que sejam musicalmente associadas, considerando, para este fim, a continuidade da próxima música com a que acabou de ser tocada [7].

\section{Avaliação}

Um dos maiores problemas na pesquisa de sistemas de recomendação para grupos é a avaliação da sua efetividade. Por exemplo, como realizar a comparação das recomendações geradas para um grupo com a verdadeira preferência dos seus membros.

Existem diversas razões que tornam os sistemas de recomendação para grupos tão difíceis de avaliar. Sendo que a combinação desses dois fatores, a seguir, tornam extremamente difícil a comparação de performance entre diferentes sistemas [15].

A primeira razão é que o critério de avaliação difere de acordo com os objetivos para os quais o sistema de recomendação esteja sendo desenvolvido. Existem situações onde o critério usado para mensurar a performance do sistema é a satisfação ou participação do usuário [55, 44, 48], enquanto em outros sistemas de recomendação para grupos, o objetivo é explorar a habilidade do sistema de mesclar perfis de usuários ou alcançar consenso entre os membros do grupo [35, 78].

O segundo motivo é a falta de base de dados públicas para realizar a avaliação. A 
maioria dos trabalhos em avaliação de sistemas de recomendação para grupos focam em experimentos com usuários reais. Neste caso, pode-se distinguir entre o trabalho usando grupos controlados que tem sido (direta ou indiretamente) questionados sobre as estratégias de agregação que eles utilizariam [44, 46] e aqueles sistemas, como Polylens e MusicFX, que tem deixado sistemas disponíveis para a comunidade de usuários. Neste caso, estudos de campo tem sido realizados para avaliar a performance do sistema.

Em uma abordagem para avaliação com a utilização de entrevista com usuários reais, existem duas opções:

- adquirir as avaliações individuais dos membros para a recomendação do grupo e depois integrar (por exemplo, somando) estas avaliações em uma pontuação como que o grupo conjuntamente atribuísse para a recomendação;

- ou adquirir diretamente a avaliação conjunta do grupo para a recomendação.

No primeiro caso, deve-se decidir como as avaliações individuais serão integradas. Este é um problema quando diferentes métodos irão produzir diferentes resultados e não há uma maneira considerada a melhor para realizar essa integração.

Outra dificuldade, comum para ambas as opções, observada por [44], é relacionada ao fato de que a satisfação de um indivíduo parece depender da satisfação dos outros membros do grupo (contágio emocional). Além disso, avaliações on-line somente são realizadas em um conjunto muito limitado de casos de teste e não podem ser usadas para testar extensivamente alternativas de algoritmos.

Uma segunda abordagem consiste em realizar uma avaliação off-line, onde grupos são escolhidos a partir dos usuários de um sistema de recomendação tradicional. Recomendações para grupos são oferecidas para membros do grupo e são avaliadas independentemente por cada membro, como no clássico caso de usuário único, pela comparação das avaliações previstas com as avaliações observados no conjunto de testes do usuário. As recomendações para grupos são geradas satisfazendo simultaneamente as preferências de todos os usuários no grupo, e, portanto, Baltrunas, Makcinskas e Ricci [8] sugerem que os resultados não podem ser tão bons na comparação com os resultados de recomendação para um único indivíduo. Os autores afirmam observar que, se utilizada esta abordagem de avaliação para testar a efetividade da recomendação para grupos, não há necessidade da avaliação conjunta dos membros do grupo para os itens recomendados. Podendo-se usar base de dados mais populares (por exemplo, MovieLens e Netflix que contém somente avaliações de usuários individuais.

${ }^{3}$ http://www.netflix.com 


\subsection{Exemplo de metodologia para avaliação off-line}

Nesta subseção, exemplificamos como efetivamente uma avaliação off-line de um sistema de recomendação para grupos pode ser conduzida. Com base nas categorizações descritas na seção 3, utilizamos as preferências individuais já conhecidas, sendo adotado um grupo passivo que não influencia como as preferências individuais são agregadas. As previsões de preferência individuais foram obtidas com um algoritmo de filtragem colaborativa [24] a partir de uma das bases de dados do MovieLens. Os itens recomendados são apresentados como uma lista de opções ao grupo. A Figura 1 ilustra a distribuição dos valores das previsões obtidas das avaliações da base do MovieLens, com média de 4,01 e desvio padrão de 0,41.

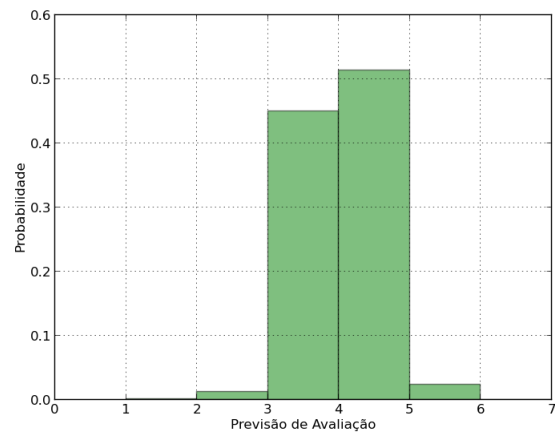

Figura 1. Histograma de previsão de avaliação.

Os itens potencialmente recomendados para cada grupo pertencem ao conjunto de itens que não foram avaliados por nenhum membro do grupo. Para tanto, eles são obtidos a partir da interseção das listas de itens de previsão de avaliação de cada usuário do grupo. Os itens pertencentes a esta lista e os valores das previsões que definem as preferências individuais dos usuários do grupo no experimento serão utilizadas nas estratégias de agregação de preferências.

Como a base do MovieLens não possui grupos definidos, faz-se necessário a formação automática dos grupos. Os usuários da base do MovieLens foram agrupados, utilizando o vetor de previsão de preferência de cada usuário, em 31 clusters com centróide aleatórios com base no método K-Means [42]. A partir desses clusters, foram selecionados aleatoriamente membros para formar dois tipos de grupos: homogêneo, contendo usuários do mesmo cluster e heterogêneo, contendo usuários de clusters diferentes. Foram ainda gerados 28 grupos desses dois tipos com 3, 5 e 7 número de membros, para se obter um nível de significância de até 0,05 na comparação das médias de satisfação e justiça das estratégias. . Dez execuções foram efetuadas. 
Tabela 5. Previsão de satisfação média para grupos homogêneos.

\begin{tabular}{lcccccccc}
\hline Size & & L.M. & Ave. & A.W.M. & M & M.P. & P.V. & Harmonic \\
\hline \multirow{2}{*}{3} & $\mu$ & 4.612 & 4.652 & 4.652 & 4.651 & 4.489 & 4.489 & 4.651 \\
& $\sigma$ & 0.100 & 0.086 & 0.086 & 0.086 & 0.117 & 0.117 & 0.085 \\
5 & $\mu$ & 4.415 & 4.457 & 4.457 & 4.457 & 4.358 & 4.358 & 4.457 \\
& $\sigma$ & 0.121 & 0.117 & 0.117 & 0.117 & 0.122 & 0.122 & 0.117 \\
7 & $\mu$ & 4.366 & 4.404 & 4.404 & 4.404 & 4.316 & 4.316 & 4.404 \\
& $\sigma$ & 0.166 & 0.167 & 0.167 & 0.167 & 0.155 & 0.155 & 0.167 \\
\hline
\end{tabular}

Propomos, como exemplo, uma estratégia de agregação baseada na Média Harmônica (Harmonic) e a comparamos com as estratégias de agregação Least Misery (L.M.), Average (Ave.), Average Without Misery (A.W.M), Multiplicative (M.), Most Pleasure (M.P.) e Plurality Voting (P.V.) em relação aos critérios de (i) satisfação e (ii) justiça (tabelas $7 \mathrm{e} 8$.

Em relação à satisfação (tabelas 5 e 6), é possível perceber que as estratégias baseadas em consenso (Average (Ave.), Average Without Misery (A.W.M), Multiplicative (M.)) obtiveram os melhores resultados (menores valores de $\mu$ ), o que é justificável devido as avaliações dos usuários estarem concentradas nos valores 4 e 5, conforme observado no histograma anterior.

Em termos de justiça, entretanto, a estratégia da média harmônica obteve o melhor resultado depois da estratégia Least Misery. Neste resultado, quanto menor os valores de variância $(\sigma)$ das preferências dos itens recomendados, maior a justiça.

Quanto à significância dos resultados da satisfação e justiça (tabelas $9 \mathrm{e} 10$, os valores em negrito referem-se a um desempenho melhor da estratégia utilizando média harmônica se comparada às demais estratégias.

\subsection{Sobre avaliação de exatidão do sistema}

Pouca atenção tem sido dada na literatura de sistemas de recomendação para grupos para a avaliação da exatidão do sistema. Esta situação claramente difere da recomendação para um único usuário. Desta forma, a avaliação da exatidão do sistema tem se tornado uma abordagem padrão [33]. É provável que uma das principais razões para isto é dar-se à dificuldade de acessar a avaliação real do grupo visto que na maioria dos casos a composição do grupo é efêmera, e não existe banco de dados com este tipo de informação [15]. Porém, em cenários reais, cada grupo avalia usando diferentes estratégias de agregação. Neste tipo 
Tabela 6. Previsão de satisfação média para grupos heterogêneos.

\begin{tabular}{lcccccccc}
\hline Size & & L.M. & Ave. & A.W.M. & M & M.P. & P.V. & Harmonic \\
\hline \multirow{2}{*}{3} & $\mu$ & 4.568 & 4.621 & 4.621 & 4.620 & 4.441 & 4.441 & 4.620 \\
& $\sigma$ & 0.100 & 0.090 & 0.090 & 0.090 & 0.135 & 0.135 & 0.090 \\
5 & $\mu$ & 4.391 & 4.436 & 4.436 & 4.436 & 4.350 & 4.350 & 4.436 \\
& $\sigma$ & 0.105 & 0.083 & 0.083 & 0.083 & 0.086 & 0.082 & 0.083 \\
7 & $\mu$ & 4.295 & 4.330 & 4.330 & 4.330 & 4.295 & 4.295 & 4.329 \\
& $\sigma$ & 0.116 & 0.130 & 0.130 & 0.130 & 0.117 & 0.117 & 0.130 \\
\hline
\end{tabular}

Tabela 7. Variância média para grupos homogêneos.

\begin{tabular}{ccccccccc}
\hline Size & & L.M. & Ave. & A.W.M. & M & M.P. & P.V. & Harmonic \\
\hline \multirow{2}{*}{3} & $\mu$ & 0.183 & 0.298 & 0.298 & 0.278 & 0.491 & 0.491 & 0.267 \\
& $\sigma$ & 0.066 & 0.093 & 0.093 & 0.884 & 0.121 & 0.121 & 0.079 \\
5 & $\mu$ & 0.222 & 0.341 & 0.341 & 0.327 & 0.444 & 0.444 & 0.319 \\
& $\sigma$ & 0.070 & 0.095 & 0.095 & 0.092 & 0.076 & 0.076 & 0.095 \\
7 & $\mu$ & 0.242 & 0.312 & 0.312 & 0.300 & 0.406 & 0.406 & 0.299 \\
& $\sigma$ & 0.083 & 0.079 & 0.079 & 0.077 & 0.056 & 0.056 & 0.078 \\
\hline
\end{tabular}

Tabela 8. Variância média para grupos heterogêneos.

\begin{tabular}{ccccccccc}
\hline Size & & L.M. & Ave. & A.W.M. & M & M.P. & P.V. & Harmonic \\
\hline \multirow{2}{*}{3} & $\mu$ & 0.159 & 0.296 & 0.296 & 0.289 & 0.530 & 0.530 & 0.275 \\
& $\sigma$ & 0.049 & 0.118 & 0.117 & 0.111 & 0.137 & 0.137 & 0.101 \\
5 & $\mu$ & 0.237 & 0.315 & 0.315 & 0.309 & 0.436 & 0.436 & 0.308 \\
& $\sigma$ & 0.056 & 0.073 & 0.072 & 0.067 & 0.091 & 0.091 & 0.067 \\
\multirow{2}{*}{7} & $\mu$ & 0.287 & 0.340 & 0.340 & 0.336 & 0.375 & 0.375 & 0.334 \\
& $\sigma$ & 0.073 & 0.066 & 0.066 & 0.067 & 0.060 & 0.060 & 0.067 \\
\hline
\end{tabular}


Tabela 9. Resultado da significância para grupos homogêneos.

\begin{tabular}{|c|c|c|c|c|c|c|c|c|c|c|c|c|}
\hline & \multicolumn{2}{|c|}{ L.M. } & \multicolumn{2}{|c|}{ Ave. } & \multicolumn{2}{|c|}{ A.W.M. } & \multicolumn{2}{|c|}{ M. } & \multicolumn{2}{|c|}{ M.P. } & \multicolumn{2}{|c|}{ P.V. } \\
\hline & $\mathrm{U}$ & $\mathrm{V}$ & $\overline{\mathrm{U}}$ & $\mathrm{V}$ & $\mathrm{U}$ & $\mathrm{V}$ & $\overline{\mathrm{U}}$ & $\mathrm{V}$ & $\mathrm{U}$ & $\mathrm{V}$ & $\overline{\mathrm{U}}$ & $\mathrm{V}$ \\
\hline 3 & 0 & 0 & 0,044 & 0,018 & 0,044 & 0,018 & 0,166 & $\mathbf{0 , 2 3 0}$ & 0 & 0 & 0 & $\mathbf{0}$ \\
\hline 5 & 0 & 0 & 0,040 & $\mathbf{0 , 0 2 5}$ & 0,040 & 0,025 & 0,162 & 0,182 & 0 & 0 & 0 & $\mathbf{0}$ \\
\hline 7 & 0 & 0 & 0,028 & 0,088 & 0,028 & $\mathbf{0 , 0 8 8}$ & 0,326 & 0,326 & 0 & 0 & 0 & 0 \\
\hline
\end{tabular}

Tabela 10. Resultado da significância para grupos heterogêneos.

\begin{tabular}{|c|c|c|c|c|c|c|c|c|c|c|c|c|}
\hline & \multicolumn{2}{|c|}{ L.M. } & \multicolumn{2}{|c|}{ Ave. } & \multicolumn{2}{|c|}{ A.W.M. } & \multicolumn{2}{|c|}{ M. } & \multicolumn{2}{|c|}{ M.P. } & \multicolumn{2}{|c|}{ P.V. } \\
\hline & $\mathrm{U}$ & $\mathrm{V}$ & $\bar{U}$ & $\mathrm{~V}$ & $\mathrm{U}$ & $\mathrm{V}$ & $\mathrm{U}$ & $\mathrm{V}$ & $\mathrm{U}$ & $\mathrm{V}$ & $\overline{\mathrm{U}}$ & $\mathrm{V}$ \\
\hline 3 & 0 & 0 & 0,132 & 0,093 & 0,132 & 0,093 & 0,169 & 0,172 & 0 & 0 & 0 & 0 \\
\hline 5 & 0 & 0 & 0,092 & 0,171 & 0,092 & 0,171 & 0,241 & $\mathbf{0 , 2 5 3}$ & 0 & 0 & 0 & 0 \\
\hline 7 & 0 & 0 & 0,171 & 0,084 & 0,171 & 0,084 & 0,326 & 0,326 & 0 & 0 & 0 & 0 \\
\hline
\end{tabular}

de situação, um algoritmo cego, que não considera como o grupo avalia um item, pode ser inapropriado para a predição de avaliações para o grupo.

\section{Conclusão}

Este tutorial tratou do estado-da-arte de sistemas de recomendação para grupos. Procuramos organizar as discussões em sessões dedicadas a cada um de seus importantes aspectos. Após introduzir o conceito de grupo, apresentamos os diferentes eixos de classificação sob os quais a literatura corrente enquadra os sistemas de recomendação para grupos, destacando-se a seguir os grandes problemas envolvidos. Grande ênfase neste tutorial foi dada as estratégias de agregação de preferência individual baseadas na teoria da Social Choice. Neste sentido, como forma de auxiliar o entendimento da metodologia para avaliação de sistemas de recomendação para grupos, propomos uma nova estratégia de agregação que considera a Média Harmônica e a utilizamos para comparar com outras já estabelecidas pela literatura. O cenário de experimentação foi claramente descrito e esperamos que esta seção contribua para que interessados no tema possam ter alguma referência de como organizar seus próprios experimentos. 


\section{Referências}

[1] G. Adomavicius and A. Tuzhilin. Toward the next generation of recommender systems: a survey of the state-of-the-art and possible extensions. IEEE Transactions on Knowledge and Data Engineering, 17(6):734-749, June 2005.

[2] Gediminas Adomavicius and Alexander Tuzhilin. Context-Aware Recommender Systems. In Francesco Ricci, Lior Rokach, Bracha Shapira, and Paul B. Kantor, editors, Recommender Systems Handbook. Springer US, Boston, MA, 2011.

[3] Esma Aïmeur, Gilles Brassard, José M Fernandez, and Flavien Serge Mani Onana. Alambic: a privacy-preserving recommender system for electronic commerce. International Journal of Information Security, 7(5):307-334, 2008.

[4] Sihem Amer-Yahia, Senjuti Basu Roy, Ashish Chawlat, Gautam Das, and Cong Yu. Group recommendation: semantics and efficiency. In Proceedings of the VLDB Endowment, volume 2, pages 754-765, August 2009.

[5] Liliana Ardissono, Anna Goy, Giovanna Petrone, Marino Segnan, and Pietro Torasso. Intrigue: Personalized Recommendation Of Tourist Attractions For Desktop And Handset Devices. APPLIED ARTIFICIAL INTELLIGENCE: Special Issue on Artificial Intelligence for Cultural Heritage and Digital Libraries, 17(8-9):687-714, 2003.

[6] Kenneth J. Arrow. Social Choice and Individual Values. Wiley, 2nd edition, 1963.

[7] Claudio Baccigalupo and Enric Plaza. A Case-Based Song Scheduler for Group Customised Radio. In Rosina O. Weber and Michael M. Richter, editors, Case-Based Reasoning Research and Development, volume 4626 of Lecture Notes in Computer Science, pages 433-448. Springer Berlin Heidelberg, Berlin, Heidelberg, 2007.

[8] Linas Baltrunas, Tadas Makcinskas, and Francesco Ricci. Group recommendations with rank aggregation and collaborative filtering. In Proceedings of the fourth ACM conference on Recommender systems - RecSys '10, page 119, New York, New York, USA, September 2010. ACM Press.

[9] Pavel Bekkerman, Sarit Kraus, and Francesco Ricci. Applying cooperative negotiation methodology to group recommendation problem. In Alexander Felfernig and Markus Zanker, editors, Proceedings of Workshop on Recommender Systems in 17th European Conference on Artificial Intelligence (ECAI 2006), pages 72-75, Riva del Garda, Italy, 2006.

[10] Shlomo Berkovsky, Yaniv Eytani, Tsvi Kuflik, and Francesco Ricci. Enhancing privacy and preserving accuracy of a distributed collaborative filtering. In Proceedings of the 2007 ACM conference on Recommender systems, pages 9-16. ACM, 2007. 
[11] Shlomo Berkovsky and Jill Freyne. Group-based recipe recommendations. In Proceedings of the fourth ACM conference on Recommender systems - RecSys '10, page 111, New York, New York, USA, September 2010. ACM Press.

[12] Ludovico Boratto and Salvatore Carta. State-of-the-Art in Group Recommendation and New Approaches for Automatic Identification of Groups . In Alessandro Soro, Eloisa Vargiu, Giuliano Armano, and Gavino Paddeu, editors, Information Retrieval and Mining in Distributed Environments, volume 324 of Studies in Computational Intelligence, pages 1-20. Springer Berlin Heidelberg, Berlin, Heidelberg, 2011.

[13] Ludovico Boratto, Salvatore Carta, Alessandro Chessa, Maurizio Agelli, and M. Laura Clemente. Group Recommendation with Automatic Identification of Users Communities. In 2009 IEEE/WIC/ACM International Joint Conference on Web Intelligence and Intelligent Agent Technology, pages 547-550. IEEE, 2009.

[14] Robin Burke. Hybrid Recommender Systems: Survey and Experiments. User Modeling and User-Adapted Interaction, 12(4):331-370, November 2002.

[15] Luis M. Campos, Juan M. Fernández-Luna, Juan F. Huete, and Miguel A. RuedaMorales. Managing uncertainty in group recommending processes. User Modeling and User-Adapted Interaction, 19(3):207-242, November 2008.

[16] L. Carvalho and W. Neto. Mypopcorn: um sistema social de recomendação de filmes. http://mypopcorn.info, 2013. [Online; accessed 19-July-2013].

[17] Lucas Augusto M.C. Carvalho and Hendrik T. Macedo. Generation of coalition structures to provide proper groups' formation in group recommender systems. In Proceedings of the 22nd international conference on World Wide Web companion, WWW '13 Companion, pages 945-950, Republic and Canton of Geneva, Switzerland, 2013. International World Wide Web Conferences Steering Committee.

[18] Lucas Augusto Montalvão Costa Carvalho and Hendrik Teixeira Macedo. Users'satisfaction in recommendation systems for groups: an approach based on noncooperative games. In Proceedings of the 22nd international conference on World Wide Web companion, WWW'13 Companion, pages 951-958, Republic and Canton of Geneva, Switzerland, 2013. International World Wide Web Conferences Steering Committee.

[19] Sílvio C. Cazella, Maria A. S. N. Nunes, and Eliseo B. Reategui. A Ciência do Palpite: Estado da Arte em Sistemas de Recomendação. In Jornada de Atualização de Informática - JAI 2010 - CSBC, pages 161-216, Rio de Janeiro, 2010.

[20] Dennis L. Chao, Justin Balthrop, and Stephanie Forrest. Adaptive radio: achieving consensus using negative preferences. In Proceedings of the 2005 international ACM 
SIGGROUP conference on Supporting group work - GROUP '05, page 120, New York, New York, USA, November 2005. ACM Press.

[21] Zunping Cheng and Neil Hurley. Effective diverse and obfuscated attacks on modelbased recommender systems. In Proceedings of the third ACM conference on Recommender systems, pages 141-148. ACM, 2009.

[22] Ingrid A Christensen and Silvia Schiaffino. Entertainment recommender systems for group of users. Expert Systems with Applications, 38(11):14127-14135, 2011.

[23] Andrew Crossen, Jay Budzik, and Kristian J. Hammond. Flytrap: intelligent group music recommendation. In Proceedings of the 7th international conference on Intelligent user interfaces - IUI '02, page 184, New York, New York, USA, January 2002. ACM Press.

[24] B. W. Dasarathy. Nearest Neighbor (NN). Los Alamitos, CA, 1991. IEEE Computer Society Press.

[25] Frederico Durao and Peter Dolog. Extending a hybrid tag-based recommender system with personalization. In Proceedings of the 2010 ACM Symposium on Applied Computing - SAC '10, page 1723, New York, New York, USA, March 2010. ACM Press.

[26] Leila Esmaeili, Mahdi Nasiri, and Behrouz Minaei-Bidgoli. Personalizing Group Recommendation to Social Network Users. In Zhiguo Gong, Xiangfeng Luo, Junjie Chen, Jingsheng Lei, and Fu Lee Wang, editors, Web Information Systems and Mining (LNCS), volume 6987 of Lecture Notes in Computer Science, pages 124-133. Springer Berlin Heidelberg, Berlin, Heidelberg, 2011.

[27] Alexander Felfernig, Christoph Zehentner, Gerald Ninaus, Harald Grabner, Walid Maalej, Dennis Pagano, Leopold Weninger, Florian Reinfrank, Liliana Ardissono, and Tsvi Kuflik. Group Decision Support for Requirements Negotiation . In Liliana Ardissono and Tsvi Kuflik, editors, Advances in User Modeling, volume 7138 of Lecture Notes in Computer Science, pages 105-116. Springer Berlin Heidelberg, Berlin, Heidelberg, 2012.

[28] A. Guilherme Galliano. Introdução à sociologia. Harper \& Row do Brasil, 1981.

[29] Inma Garcia, Sergio Pajares, Laura Sebastia, and Eva Onaindia. Preference elicitation techniques for group recommender systems. Information Sciences, 189:155-175, April 2012 .

[30] Inma Garcia, Laura Sebastia, and Eva Onaindia. On the design of individual and group recommender systems for tourism. Expert Systems with Applications, 38(6):7683-7692, 2011. 
[31] Inma Garcia, Laura Sebastia, Eva Onaindia, and Cesar Guzman. A Group Recommender System for Tourist Activities. In Tommaso Noia and Francesco Buccafurri, editors, E-Commerce and Web Technologies, volume 5692 of Lecture Notes in Computer Science, pages 26-37. Springer Berlin Heidelberg, Berlin, Heidelberg, 2009.

[32] Inma Garcia, Laura Sebastia, Sergio Pajares, and Eva Onaindia. Approaches to Preference Elicitation for Group Recommendation . In Beniamino Murgante, Osvaldo Gervasi, Andrés Iglesias, David Taniar, and Bernady O. Apduhan, editors, Computational Science and Its Applications - ICCSA 2011, volume 6786 of Lecture Notes in Computer Science, pages 547-561. Springer Berlin Heidelberg, Berlin, Heidelberg, 2011.

[33] Jonathan L. Herlocker, Joseph A. Konstan, Loren G. Terveen, and John T. Riedl. Evaluating collaborative filtering recommender systems. ACM Transactions on Information Systems, 22(1):5-53, 2004.

[34] Rong $\mathrm{Hu}$ and Pearl Pu. Using Personality Information in Collaborative Filtering for New Users. In 2nd ACM RecSys'10 Workshop on Recommender Systems and the Social Web, pages 17-24, New York, NY, USA, 2010.

[35] Anthony Jameson. More than the sum of its members: challenges for group recommender systems. In Proceedings of the working conference on Advanced visual interfaces $A V I$ '04, page 48, New York, New York, USA, May 2004. ACM Press.

[36] Anthony Jameson and Barry Smyth. Recommendation to Groups. In Peter Brusilovsky, Alfred Kobsa, and Wolfgang Nejdl, editors, The Adaptive Web (LNCS), volume 4321 of Lecture Notes in Computer Science, pages 596-627. Springer Berlin Heidelberg, Berlin, Heidelberg, 2007.

[37] Judy Kay and William Niu. Adapting Information Delivery to Groups of People. In Workshop on New Technologies for Personalized Information Access in 10th International Conference on User Modeling (UM'2005), 2005.

[38] Jae Kyeong Kim, Hyea Kyeong Kim, Hee Young Oh, and Young U. Ryu. A group recommendation system for online communities. International Journal of Information Management, 30(3):212-219, June 2010.

[39] Ricardo Lage, Frederico Durao, and Peter Dolog. Towards effective group recommendations for microblogging users. In Proceedings of the 27th Annual ACM Symposium on Applied Computing - SAC '12, page 923, New York, New York, USA, March 2012. ACM Press.

[40] H Lieberman. Let's browse: a collaborative browsing agent. Knowledge-Based Systems, 12(8):427-431, December 1999. 
[41] Kuei-Hong Lin, Yu-Shian Chiu, and Jia-Sin Chen. An adaptive correlation-based group recommendation system . In Intelligent Signal Processing and Communications Systems (ISPACS), 2011 International Symposium on, Chiang Mai, 2011.

[42] J. B. MacQueen. Some methods for classification and analysis of multivariate observations. In Proceedings of the Fifth Symposium on Math, Statistics, and Probability, pages 281 - 297. University of California Press., 1967.

[43] Monika Mandl, Alexander Felfernig, Erich Teppan, and Monika Schubert. Consumer decision making in knowledge-based recommendation. Journal of Intelligent Information Systems, 37(1):1-22, 2011.

[44] Judith Masthoff. Group Modeling: Selecting a Sequence of Television Items to Suit a Group of Viewers. User Modeling and User-Adapted Interaction, 14(1):37-85, February 2004.

[45] Judith Masthoff. Group recommender systems: combining individual models. In Francesco Ricci, Lior Rokach, Bracha Shapira, and Paul B. Kantor, editors, Recommender Systems Handbook, page 677. Springer US, Boston, MA, 2011.

[46] Judith Masthoff and Albert Gatt. In pursuit of satisfaction and the prevention of embarrassment: affective state in group recommender systems. User Modeling and UserAdapted Interaction, 16(3-4):281-319, August 2006.

[47] Joseph F. McCarthy. Pocket Restaurant Finder: A situated recommender system for groups. In Proceedings of the Workshop on Mobile Ad-Hoc Communication at the 2002 ACM Conference on Human Factors in Computer Systems, Minneapolis, 2002.

[48] Joseph F. McCarthy and Theodore D. Anagnost. MusicFX: an arbiter of group preferences for computer supported collaborative workouts. In Proceedings of the 1998 ACM conference on Computer supported cooperative work - CSCW'98, pages 363-372, New York, New York, USA, November 1998. ACM Press.

[49] Joseph F. McCarthy, Tony Costa, and Edy Liongosari. UniCast, OutCast \& GroupCast: Three Steps Toward Ubiquitous, Peripheral Displays. In Gregory D. Abowd, Barry Brumitt, and Steven Shafer, editors, Ubicomp 2001: Ubiquitous Computing, volume 2201 of Lecture Notes in Computer Science, pages 332-345. Springer Berlin Heidelberg, Berlin, Heidelberg, October 2001.

[50] Kevin McCarthy, Lorraine McGinty, Barry Smyth, and Maria Salamó. The needs of the many: A case-based group recommender system. . In Thomas R. Roth-Berghofer, Mehmet H. Göker, and H. Altay Güvenir, editors, Advances in Case-Based Reasoning, volume 4106 of Lecture Notes in Computer Science, pages 196-210. Springer Berlin Heidelberg, Berlin, Heidelberg, 2006. 
[51] Kevin McCarthy, Maria Salamó, Lorcan Coyle, Lorraine McGinty, Barry Smyth, and Paddy Nixon. Cats: A synchronous approach to collaborative group recommendation. Proceedings of FLAIRS Conference'2006, 2006.

[52] Frank McSherry and Ilya Mironov. Differentially private recommender systems: building privacy into the net. In Proceedings of the 15th ACM SIGKDD international conference on Knowledge discovery and data mining, pages 627-636. ACM, 2009.

[53] John Von Neumann and O Morgenstern. Theory of Games and Economic Behavior. Princeton University Press, Princeton, NJ, 2nd edition, 1947.

[54] Maria Augusta Silveira Netto Nunes. Recommender Systems based on Personality Traits: Could human psychological aspects influence the computer decision-making process?, volume 1. VDM Verlag Dr. Müller, Berlin, 1 edition, 2009.

[55] Mark O’Connor, Dan Cosley, Joseph A. Konstan, and John T. Riedl. PolyLens: A Recommender System for Groups of Users. In Wolfgang Prinz, Matthias Jarke, Yvonne Rogers, Kjeld Schmidt, and Volker Wulf, editors, ECSCW 2001, pages 199_ 218. Kluwer Academic Publishers, Dordrecht, 2002.

[56] A. A. OLIVEIRA, E. D. M. ORDONEZ, and C. C. J. ALMEIDA. Anais 2012 workshop tecnologias da informação e comunicação nos grandes eventos esportivos do brasil. volume 1, page 133p. UFS e Sergipetec, 2012.

[57] Michael J. Pazzani and Daniel Billsus. Content-Based Recommendation Systems. In Peter Brusilovsky, Alfred Kobsa, and Wolfgang Nejdl, editors, The Adaptive Web, volume 4321 of Lecture Notes in Computer Science. Springer Berlin Heidelberg, Berlin, Heidelberg, 2007.

[58] David M. Pennock, Eric Horvitz, and C. Lee Giles. Social choice theory and recommender systems: Analysis of the axiomatic foundations of collaborative filtering. In Proc. 17th AAAI, 2000.

[59] Sebastiano Pizzutilo, Berardina De Carolis, Giovanni Cozzolongo, and Francesco Ambruoso. Group modeling in a public space: methods, techniques, experiences. In AIC'05 Proceedings of the 5th WSEAS International Conference on Applied Informatics and Communications, pages 175-180, September 2005.

[60] Gheorghe Popescu. Preference Aggregation in Group and Social Recommender Systems. Technical report, EPFL, Lousanna, Switzeland, 2011.

[61] Gheorghe Popescu and Pearl Pu. Probabilistic game theoretic algorithms for group recommender systems. In ACM RecSys, Workshop on Music Recommendation and Discovery, pages 30-33, Chicago, IL, USA, 2011. ACM. 
[62] L. Quijano-Sanchez, J.A. Recio-Garcia, and B. Diaz-Agudo. HappyMovie: A Facebook Application for Recommending Movies to Groups . In Tools with Artificial Intelligence (ICTAI), 2011 23rd IEEE International Conference on, pages 239 - 244, Boca Raton, FL, 2011.

[63] Lara Quijano-Sánchez, Derek Bridge, Belén Díaz-Agudo, and Juan A. Recio-García. A Case-Based Solution to the Cold-Start Problem in Group Recommenders. In Belén Díaz Agudo and Ian Watson, editors, Case-Based Reasoning Research and Development, volume 7466 of Lecture Notes in Computer Science. Springer Berlin Heidelberg, Berlin, Heidelberg, 2012.

[64] Lara Quijano-Sánchez, Juan Recio Garcia, and Díaz-Agudo. Personality and Social Trust in Group Recommendations. In 2010 22nd International Conference on Tools with Artificial Intelligence, pages 121-126, Berlin, Heidelberg, 2010.

[65] Lara Quijano-Sánchez, Juan Recio Garcia, Belén Díaz-Agudo, Ashwin Ram, and Nirmalie Wiratunga. Using Personality to Create Alliances in Group Recommender Systems . In Ashwin Ram and Nirmalie Wiratunga, editors, Case-Based Reasoning Research and Development, volume 6880 of Lecture Notes in Computer Science, pages 226-240. Springer Berlin Heidelberg, Berlin, Heidelberg, 2011.

[66] Juan A. Recio-Garcia, Guillermo Jimenez-Diaz, Antonio A. Sanchez-Ruiz, and Belen Diaz-Agudo. Personality aware recommendations to groups. In Proceedings of the third ACM conference on Recommender systems - RecSys '09, page 325, New York, New York, USA, October 2009. ACM Press.

[67] Paul Resnick and Hal R. Varian. Recommender systems. Communications of the ACM, 40(3):56-58, 1997.

[68] Andrew I. Schein, Alexandrin Popescul, Lyle H. Ungar, and David M. Pennock. Methods and metrics for cold-start recommendations. In Proceedings of the 25th annual international ACM SIGIR conference on Research and development in information retrieval - SIGIR '02, page 253, New York, New York, USA, August 2002. ACM Press.

[69] Amartya Sen. Rationality and Social Choice. In Handbook of Mathematical Economics, volume 3. Elsevier Science, 1995.

[70] Christophe Senot, Dimitre Kostadinov, Makram Bouzid, Jérôme Picault, and Armen Aghasaryan. Evaluation of Group Profiling Strategies. In Proceedings of the Twenty-Second International Joint Conference on Artificial Intelligence, pages 2728 -2733, Barcelona, 2011. the Association for the Advancement of Artificial Intelligence (AAAI). 
[71] Christophe Senot, Dimitre Kostadinov, Makram Bouzid, Jérôme Picault, Armen Aghasaryan, and Cédric Bernier. Analysis of Strategies for Building Group Profiles. In Paul Bra, Alfred Kobsa, and David Chin, editors, User Modeling, Adaptation, and Personalization, volume 6075 of Lecture Notes in Computer Science, pages 40-51. Springer Berlin Heidelberg, Berlin, Heidelberg, 2010.

[72] K Shyong, Dan Frankowski, John Riedl, et al. Do you trust your recommendations? an exploration of security and privacy issues in recommender systems. In Emerging Trends in Information and Communication Security, pages 14-29. Springer, 2006.

[73] Barry Smyth, Evelyn Balfe, Jill Freyne, Peter Briggs, Maurice Coyle, and Oisin Boydell. Exploiting Query Repetition and Regularity in an Adaptive Community-Based Web Search Engine. User Modeling and User-Adapted Interaction, 14(5):383-423, April 2005.

[74] Rui Ping Song, Bo Wang, Guo Ming Huang, Qi Dong Liu, Rong Jing Hu, and Rui Sheng Zhang. A hybrid recommender algorithm based on an improved similarity method. Applied Mechanics and Materials, 475:978-982, 2014.

[75] David Sprague, Fuqu Wu, and Melanie Tory. Music selection using the Party Vote democratic jukebox. In Proceedings of the working conference on Advanced visual interfaces - $A V I$ '08, page 433, New York, New York, USA, May 2008. ACM Press.

[76] Fabiana Vernero. Double-Sided Recommendations: A Novel Framework for Recommender Systems. In Roberto Pirrone and Filippo Sorbello, editors, AI*IA 2011: Artificial Intelligence Around Man and Beyond, volume 6934 of Lecture Notes in Computer Science, pages 262-273. Springer Berlin Heidelberg, Berlin, Heidelberg, 2011.

[77] Jing Wang, Zhijing Liu, and Hui Zhao. Group Recommendation Based on the PageRank. Journal of Networks, 7:2019-2024, 2012.

[78] Zhiwen Yu, Xingshe Zhou, Yanbin Hao, and Jianhua Gu. TV Program Recommendation for Multiple Viewers Based on user Profile Merging. User Modeling and UserAdapted Interaction, 16(1):63-82, June 2006.

[79] Elena Zheleva and Lise Getoor. Privacy in social networks: A survey. In Social Network Data Analytics, pages 277-306. Springer, 2011.

[80] Yu Zhiwen, Zhou Xingshe, and Zhang Daqing. An adaptive in-vehicle multimedia recommender for group users . In Vehicular Technology Conference, 2005. VTC 2005Spring. 2005 IEEE 61st, page 2800, 2005. 